\title{
COMPARATIVE STUDY OF FUNCTIONALLY GRADED MATERIAL MODELS FOR STRUCTURAL DESIGN OF THIN-WALLED BLADES
}

\author{
Touraj FARSADI * \\ Aerospace Engineering Department, Adana Alparslan Türkeş Science and Technology University, Adana, Turkey
}

\begin{abstract}
In this work, three theories of Functionally Graded Material (FGM) are compared for structural dynamic and static performance of the thin-walled rotating blade. For this purpose, the pretwisted Thin Wall Rotating Beam (TWRB) with a fixed angular velocity is considered. The goal is to find the desirable FG model with improved free vibration, static deformation, and buckling behavior of the FGM blades. The Euler-Lagrange equations of motion of the energetic system are extracted utilizing Hamilton's principle. The Extended Galerkin`s Method (EGM) is used to solve the governing equation of motions. The effects of some parameters, such as the FGM models, angular velocity, and pretwist angle on the mechanical behavior of the FG beams are studied.
\end{abstract}

Keywords: Thin-walled blade; Functionally graded material; Structural dynamics

\section{INTRODUCTION}

The Functionally Graded Material (FGM) is an inhomogeneous material, composed of two (or more) materials, organized to have a smooth gradation in the desired direction. An example of an FGM part is a metal-to-ceramic beam, in which the two materials cancel out each other's weaknesses and enhance each other's strengths. Ceramic by itself is hard and chemically and thermally resistant, but it's also brittle with low impact toughness. Metal, on the other hand, is strong with high impact toughness, but it's easily corroded by strong acids and bases and its mechanical performance suffers when heated. Put the two materials together, though, and they become virtually indestructible. Thanks to smoothly and continuously varying material properties which give superior mechanical and thermal characteristics to FGMs and provides sufficient strength and toughness to withstand extreme mechanical and thermal loading conditions [1-4]. Applications related to FG materials are outlined by Mahamood and Akinlabi [5] who give a broad review of the evolution of FGMs.

Thin-walled Rotating beams (TWRB) and blades made of FGMs are important structural components. They play a substantial role in engineering structures owing to their impressive strength-to-weight ratio, extended fatigue life, and other outstanding engineering properties. Librescu et. al. [6] considered difficulties related to elastic modeling and performance of TWBs made of FGM. They utilized a powerlaw formulation for the conformation of the material phases distributed in the wall thickness direction. Elastic modeling and dynamics of turbomachinery TWRB made of FG ceramic/metal materials are studied by Oh et. al [7]. Bahaadini and Saidi [8] study the aeroelastic behavior of FG nanocomposite TWRBs made of GPLs exposed to supersonic aerodynamic flow. The differential governing equations of motion are deduced considering the thin-walled Timoshenko beam. Piovan and Machado [9] did the dynamic analysis of the FG TWB exposed to the axial force by considering the non-linear straindisplacement theory. Latalskia and Warminskia [10] applied Galerkin's technique to achieve formulations of coupled bending-shear-twist dynamics of the TWRB made of composite material. Sina

*Corresponding Author: tfarsadi@atu.edu.tr

Received: 25.01.2021 Published: 29.09.2021 
et. al. [11] examined the angular velocity effect in the free vibration of composite TWB. Oh and Yoo [12] proposed a structural model to investigate the vibration performance of a rotary FG blade with geometrical non-uniformities using Rayleigh-Ritz assumed mode technique in combination with Kane's method. Aeroelastic performance of rotating FGM TWBs under supersonic airflow was considered by Fazelzadeh et. al. [13, 14]. The effects of pretwist on the chordwise and flapwise bending vibrations of thin-walled FG beams in a high-temperature field are addressed by Oh et al [15, 16]. Maalawi [17] developed a method for designing effective forms of FGM bars with optimized frequencies.

In this paper, a blade with three FG distribution functions exposed to centrifugal loading owing to fixed angular velocity is considered. The free vibration, static, and buckling performance of turbomachinery TWRB made of various models of functionally graded ceramic/metal materials are compared. Three formulas concerning material gradation through FGMs include Power-law, Trigonometric, and ViolaTornabene three-parameter models. The recommended models have succeeded in finding the desirable solutions representing substantial improvements in the targeted design objective. So far, no study has been reported considering the comparative study of functionally graded TWRB with various FG models. The present work aims to explore the effect of property gradation on the frequencies, static deflections, and critical buckling load of the TWRB.

\section{GRADATION RELATION}

Figure 1 shows a schematic description of a blade with the interior main load-carrying spar box beam structure. The latter is made of FGMs which can be suitable candidates for aeronautical structures. They are believed to be an alternative solution for a certain class of aerospace structures exposed to highstress environments.

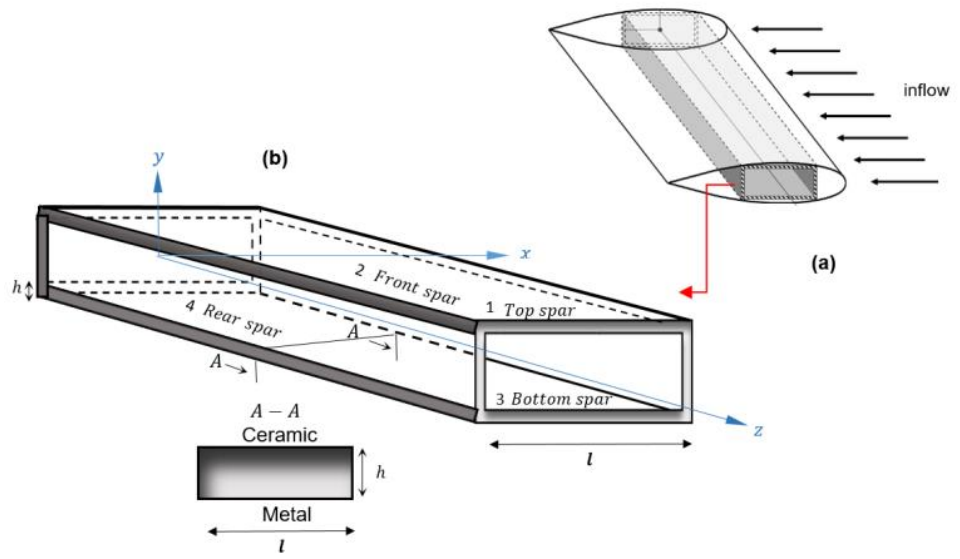

(c)

Figure 1. Schematic description of the a) blade and b) torque box of the blade modeled as TWRB made of c) FG ceramic/metal materials

An FGM is typically a mixture of two types of materials that have a steady change of properties from one side of the specimen to the other side. Considering an FG beam, the Elastic Modulus $E$, Poisson's ratio $v$, and density $\rho$ can be stated through a distribution function as follows,

$$
\begin{aligned}
E(z) & =\left[E_{T}-E_{B}\right] V_{f}+E_{B} \\
v(z) & =\left[v_{T}-v_{B}\right] V_{f}+v_{B} \\
\rho(z) & =\left[\rho_{T}-\rho_{B}\right] V_{f}+\rho_{B}
\end{aligned}
$$

Where $V_{f}$ denotes applicable formulas regarding material gradation through FGMs. Sub-indices $T$ and $B$ represent material identifications positioned at the top and bottom walls of the beam, respectively. 
The parameter $n$ is the coordinate axis in thickness direction. In this work, the top and bottom surface materials are ceramic and metal, respectively. The zero volume fraction exponent $k$ corresponds to a fully ceramic configuration. The common FG structures contain two materials whose volume fractions change gradually. Thus, the functions working for adjusting the volume fraction exponent of material parts of the structures are important. The studied models for material gradation through FGMs in the present study are given as,

Power-law model:

$$
V_{f}=\left[\frac{1}{2}+\frac{n}{h}\right]^{k}
$$

Trigonometric model:

$$
V_{f}=\left[\sin \left[\frac{1}{2}+\frac{n}{h}\right]^{k}\right]^{2}
$$

Viola-Tornabene three-parameter model:

$$
V_{f}=\left[\frac{1}{2}-\frac{n}{h}+b\left[\frac{1}{2}+\frac{n}{h}\right]^{c}\right]^{k}
$$

Where $k$ represents the volume fraction exponent. $b$ and $c$ are coefficients that affect the material distribution.

\section{STRUCTURAL MODEL}

\subsection{Basic Assumptions}

Figure 2 demonstrates a graphic description of the FG TWRB. A rotary TWB with length $L$ and wall beam thickness $h$. The blade is connected to a rigid hub of radius $R_{0}$. It is presumed that the beam rotates about an axis perpendicular to the longitudinal axis of the blade with a fixed angular velocity $\Omega$. The origin of the rotating axis system $(x, y, z)$ is positioned at the blade root at the distance of $R_{0}$ from the rotary axis. Similarly, the curvilinear coordinate system $\left(x^{p}, y^{p}, z^{p}\right)$ is introduced besides the rotating coordinates $(x, y, z)$, where $x_{p}, y_{p}$ and $z_{p}$ represent the principal axes of the blade cross-section. In the pretwisted model of the TWB, the cross-section form is preserved, and rotary inertia and transverse shear, are involved in the beam structure. Because of the pretwist, the inertia and stiffness expressions of the TWB become twist angle $\left(\beta(z)=\beta_{0} z / L\right)$ dependent. Where $\beta_{0}$ is the pretwist angle at the tip section of the beam. The following transformation relationships are applied to connect the two coordinate systems, [18],

$$
\begin{gathered}
x=x^{p}(s) \cos \beta(z)-y^{p}(s) \sin \beta(z) \\
y=x^{p}(s) \sin \beta(z)+y^{p}(s) \cos \beta(z) \\
z=z^{p}
\end{gathered}
$$

The local coordinate system $(n, s, z)$ is located at the midplane of the cross-section of the TWB. $n$ is the coordinate axis normal to the tangential axis and $s$ is the local coordinate axis parallel to the middle surface. It should be noted that Farsadi et. al. $[18,19]$ discuss the basic assumptions in deriving the governing equations of the present beam model. 


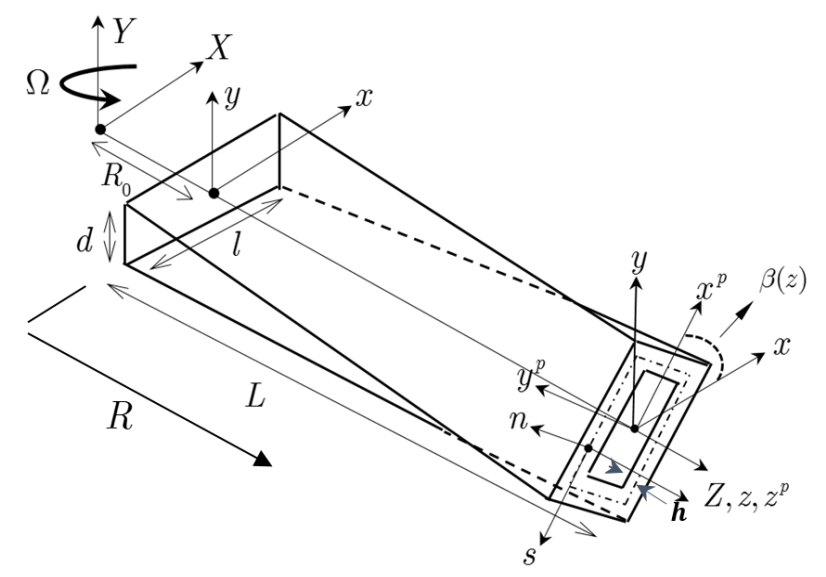

Figure 2. Description of the torque box of the blade modeled as rotating non-uniform TWB

\subsection{Kinematics}

The displacement domain of the TWB model is considered to be, [20]

$$
\begin{gathered}
u(x, y, z, t)=u_{0}(z, t) \\
v(x, y, z, t)=v_{0}(z, t) \\
w(x, y, z, t)=w_{0}(z, t)+\left(x+n \frac{d y}{d s}\right) \theta_{y}(z, t)+\left(y-n \frac{d x}{d s}\right) \theta_{x}(z, t)
\end{gathered}
$$

Where, $u_{0}, v_{0}, w_{0}$ are the TWRB shear center translations in the $x, y$ and $z$ directions, respectively. $\theta_{x}$, $\theta_{y}$ are the rotations about the $x$ and $y$ axes. The following strain-displacement relations are stated as,

$$
\varepsilon_{z z}=\frac{\partial w}{\partial z}, \quad \gamma_{s z}=\left(\theta_{y}+u_{0}^{\prime}\right) \frac{d x}{d s}+\left(\theta_{x}+v_{0}^{\prime}\right) \frac{d y}{d s}, \quad \gamma_{n z}=\left(\theta_{x}+v_{0}^{\prime}\right) \frac{d y}{d s}-\left(\theta_{y}+u_{0}^{\prime}\right) \frac{d x}{d s}
$$

\subsection{Dynamic Governing Equation of Motions}

The dynamic governing differential equations of motion can be extracted from Hamilton's principle that is given as,

$$
\begin{gathered}
\int_{t_{1}}^{t_{2}}(\delta T-\delta V+\delta W) d t=0 \\
\delta u_{0}=\delta v_{0}=\delta w_{0}=\delta \theta_{x}=\delta \theta_{y}=0
\end{gathered}
$$

where, $\delta T, \delta V, \delta W$ are the variational form of the blade's kinetic, strain energies, and the work done by external loads, respectively. For the free vibration analysis, the external forces are not considered in the equations of motion. The variation of the kinetic energy is stated as, 
$\delta T$

$$
=\int_{t_{1}}^{t_{2}} d t \int_{0}^{L}-\left\{\begin{array}{c}
b_{1}\left[\ddot{u}_{0}+2 \dot{w}_{0} \Omega-u_{0} \Omega^{2}\right] \delta u_{0}+b_{1} \ddot{v}_{0} \delta v_{0}+b_{1}\left[\ddot{w}_{0}-2 \dot{u}_{0} \Omega-\left[R_{0}+z+w_{0}\right] \Omega^{2}\right] \delta w_{0} \\
+\left[\left[b_{4}+b_{14}\right]\left[\ddot{\theta}_{x}-\Omega^{2} \theta_{x}\right]+\left[b_{6}-b_{13}\right]\left[\ddot{\theta}_{y}-\Omega^{2} \theta_{x}\right]\right] \delta \theta_{x} \\
+\left[\left[b_{5}+b_{15}\right]\left[\ddot{\theta}_{y}-\Omega^{2} \theta_{y}\right]+\left[b_{6}-b_{13}\right]\left[\ddot{\theta}_{x}-\Omega^{2} \theta_{x}\right]\right] \delta \theta_{y}
\end{array}\right\} d z
$$

The variation form of potential energy associated with the beam structure is defined as,

$$
\begin{aligned}
\delta V= & -\int_{0}^{L}\left\{\begin{array}{c}
T^{\prime}{ }_{z} \delta w_{0}+\left[M^{\prime}{ }_{x}-Q_{y}\right] \delta \theta_{x}+\left[M^{\prime}{ }_{y}-Q_{x}\right] \delta \theta_{y}+\left[Q^{\prime}{ }_{x}+\left[T_{z} u^{\prime}{ }_{z}\right]^{\prime}\right] \delta u_{0} \\
+\left[Q^{\prime}{ }_{y}+\left[T_{z} v_{z}{ }_{z}\right]^{\prime}\right] \delta v_{0}
\end{array}\right\} d z \\
& +\left.\left[T_{z} \delta w_{0}+M_{x} \delta \theta_{x}+M_{y} \delta \theta_{y}+\left[Q_{x}+T_{z} u^{\prime}{ }_{z}\right] \delta u_{0}+\left[Q_{y}+T_{z} v^{\prime}{ }_{z}\right] \delta v_{0}\right]\right|_{0} ^{L}
\end{aligned}
$$

where prime stands for the partial derivative with respect to $z . T_{z}, Q_{x}, Q_{y}, M_{x}$ and $M_{y}$ are the axial force, the chord-wise and flap-wise shear forces, the flapwise bending moment (moment about x-direction), and chordwise bending moment (moment about y-direction), respectively.

Virtual work of an external force is written as,

$$
\delta W=\int_{0}^{L}\left[p_{x} \delta u_{0}+p_{y} \delta v_{0}\right] d z
$$

Substituting Eqs. (9)-(11) into Hamilton's principle, and integrating over the beam thickness and applying the independent properties of variations, together with setting the terms of $\delta u_{0}, \delta v_{0}, \delta w_{0}, \delta \theta_{x}$, $\delta \theta_{y}$ to zero result in the equations of motion as,

$$
\begin{gathered}
\delta u_{0}: \underbrace{T_{z}}_{T_{z} u_{0}}+\left[a_{22}(z)\left[u_{0}^{\prime}+\theta_{y}\right]+a_{23}(z)\left[v^{\prime}{ }_{0}+\theta_{x}\right]\right]^{\prime}-b_{1}(z) \ddot{u}_{0}+ \\
b_{1}(z) \Omega^{2} u_{0}+\Omega^{2}\left[R(z) u_{0}^{\prime}\right]^{\prime}-2 b_{1}(z) \Omega \dot{w}_{0}+p_{x}=0 \\
\delta v_{0}: \underbrace{T_{z} v_{0}^{\prime \prime}}+\left[a_{33}(z)\left[v^{\prime}{ }_{0}+\theta_{x}\right]+a_{23}(z)\left[u^{\prime}{ }_{0}+\theta_{y}\right]\right]^{\prime}-b_{1}(z) \ddot{v}_{0}+\Omega^{2}\left[R(z) v^{\prime}{ }_{0}\right]^{\prime}+p_{y}=0 \\
\delta w_{0}:\left[a_{11}(z) w^{\prime}{ }_{0}\right]^{\prime}-b_{1}(z) \ddot{w}_{0}+b_{1}(z) \Omega^{2}\left[R_{0}+z+w_{0}\right]+2 b_{1}(z) \Omega \dot{u}_{0}=0 \\
\delta \theta_{x}:\left[a_{55}(z) \theta^{\prime}{ }_{x}+a_{54}(z) \theta^{\prime}\right]^{\prime}-a_{33}(z)\left[v^{\prime}{ }_{0}+\theta_{x}\right]-a_{32}(z)\left[u^{\prime}{ }_{0}+\theta_{y}\right] \\
-\left[b_{4}(z)+b_{14}(z)\right]\left[\ddot{\theta}_{x}-\Omega^{2} \theta_{x}\right]-\left[b_{6}(z)-b_{13}(z)\right]\left[\ddot{\theta}_{y}-\Omega^{2} \theta_{y}\right]=0 \\
\delta \theta_{y}:\left[a_{44}(z) \theta^{\prime}{ }_{y}+a_{45}(z) \theta^{\prime}{ }_{x}\right]^{\prime}-a_{22}(z)\left[u_{0}^{\prime}+\theta_{y}\right]-a_{23}(z)\left[v^{\prime}{ }_{0}+\theta_{x}\right]- \\
{\left[b_{5}(z)+b_{15}(z)\right]\left[\ddot{\theta}_{y}-\Omega^{2} \theta_{y}\right]-\left[b_{6}(z)-b_{13}(z)\right]\left[\ddot{\theta}_{x}-\Omega^{2} \theta_{x}\right]=0}
\end{gathered}
$$

In the above relations, the underlined terms are specific to the buckling equations. When these terms are omitted, the remaining parts constitute the linear equations of motion for the beam structure in terms of force expressions. The description of the stiffness coefficients is made in Table 1.

Table 1. Description of stiffness terms

\begin{tabular}{|c|c|c|c|}
\hline$a_{11}$ & Extensional & $a_{22}$ & Chordwise shear \\
\hline$a_{33}$ & Flapwise shear & $a_{44}$ & Chordwise bending \\
\hline$a_{55}$ & Flapwise bending & $a_{23}$ & Chordwise shear - Flpawise shear \\
\hline$a_{45}$ & Chordwise bending - Flapwise bending & & \\
\hline
\end{tabular}


Herein, the coefficients $b_{i}$ and $a_{i j}$ represent the reduced mass and stiffness coefficients, respectively, which are introduced in Ref [19]. Furthermore, $R(z)$ denotes the centrifugal force parameter which is expressed as,

$$
R(z)=\left[R_{0}[L-z]+\frac{1}{2}\left[L^{2}-z^{2}\right]\right]
$$

\section{SOLUTION METHODOLOGY}

\subsection{Free and Forced Vibration}

The Extended Galerkin Method (EGM) in combination with the separation of variables technique [21] is applied to solve the governing partial differential equation of motion for the natural frequencies and the related mode shapes. The linear combination of the shape functions is applied. By gradually increasing the number of terms in the series the convergence is reached. In the Extended Galerkin Strategy, the unknown displacement variables are estimated in series form by,

$$
\begin{gathered}
u_{0}(z, t)=\sum_{i=1}^{N} \eta_{i}^{u}(t) \psi_{i}^{u}(z), \quad v_{0}(z, t)=\sum_{i=1}^{N} \eta_{i}^{v}(t) \psi_{i}^{v}(z), \quad w_{0}(z, t)=\sum_{i=1}^{N} \eta_{i}^{w}(t) \psi_{i}^{w}(z), \\
\theta_{x}(z, t)=\sum_{i=1}^{N} \eta_{i}^{x}(t) \psi_{i}^{x}(z), \quad \theta_{y}(z, t)=\sum_{i=1}^{N} \eta_{i}^{y}(t) \psi_{i}^{y}(z),
\end{gathered}
$$

where the trial functions $\psi^{u}, \psi^{v}, \psi^{w}, \psi^{x}, \psi^{y}$ have to be rationally selected to fulfill the essential boundary conditions. $N$ is selected properly to attain the convergence. Trial functions for all degrees of freedom are taken in a polynomial form as $\left(\psi_{i}=z^{i}, i=1,2, \ldots, N\right)$. Seven trial functions $(N=7)$, which is found to be sufficient for the convergence of the aeroelastic solution, are applied to satisfy the boundary conditions of clamped-free blade structure. The time-dependent variables in the state vector form are defined by Equation (15).

$$
\eta=\left[\left[\begin{array}{lll}
\left.\eta^{u}\right]^{T} & {\left[\eta^{v}\right.}
\end{array}\right]^{T} \quad\left[\eta^{w}\right]^{T} \quad\left[\eta^{x}\right]^{T} \quad\left[\eta^{y}\right]^{T}\right]^{T}
$$

Inserting Equation (14) into Equation (9), Equation (10), and Equation (11) one can write the kinetic energy, potential energy, and work done by external force in the linear and discrete form, respectively.

$$
T=\frac{1}{2} \dot{\eta}^{T} M_{s} \dot{\eta}, \quad V=\frac{1}{2} \eta^{T} K_{s} \eta, \quad \delta W=Q^{T} \delta \eta
$$

Where $M_{S}, K_{s}$ are the structural mass and stiffness matrices and,

$$
Q=\int_{0}^{L}\left[\left[p_{x} \psi^{u^{T}}+p_{y} \psi^{v T}\right]^{T}\right] d z
$$

Introducing Equation (16) and Equation (17) in Hamilton principle, integrating with respect to time, and knowing $\delta \eta=0$ at $t=t_{1}, t_{2}$ one can obtain the discrete governing equations of motion as,

$$
\left[M_{S}\right] \vec{\eta}+\left[K_{S}\right] \vec{\eta}=\vec{Q}
$$

The analytical integral calculations are performed to extract the mass and stiffness matrices.

To perform free vibration analysis, the force vector on the right-hand side is ignored and Equation (18) is solved for the eigenvalues and the corresponding eigenvectors accordingly. 


\subsection{Buckling Analysis}

The set of linear equations resulting from the application of the Galerkin method rearranged to form a homogeneous matrix equation of an eigenvalue problem which upon numeric solution, gives the critical value of the axial load corresponding to the buckling of the structure. Following the same solution technique introduced in the solution methodology section 4.1, the final linear equations resulting from the application of the EGM is posed as an eigenvalue problem given by Equation (19).

$$
\left[\left[K_{s}\right]-T_{z}\left[K_{b}\right]\right] \bar{X}=0
$$

In Equation (19), $K_{s}$ is the stiffness matrix resulting from the application of the EGM and $K_{b}$ is the buckling stiffness matrix. $\bar{X}$ is the vector of unknown constants and $T_{z}$ is the eigenvalue that is the minimum of which corresponds to the critical axial buckling load. The dimensionless critical buckling load factor $\bar{P}$ is defined by Equation (20). The critical buckling load factor $\bar{P}$ is another metric for the evaluation of structural performance.

$$
\bar{P}=\frac{T_{Z} L^{2}}{h d^{3} E}
$$

\section{RESULTS AND DISCUSSION}

\subsection{Validation of the Computer Code}

The results of Librescu et al. [6] are used to compare the variations of the three natural frequencies of the rotating and pretwisted TWB made of FGM. The results of the validation are given in

Table 2. The considered TWRB has a rectangular uniform cross-section with the geometrical and material characteristics given in Table 3. Note that " $m$ " and " $c$ " subscripts stand for metal (SUS304) and ceramic $\left(\mathrm{Si}_{3} \mathrm{~N}_{4}\right)$ materials, respectively. It can be detected that an accurate agreement exists among the numerical results of the present work and those stated in Ref [6]. In the numerical simulations, the dimensionless parameters given in Equation (21) are considered, where $b_{1}$ and $a_{55}$ are the sectional properties of the uniformed non-pretwisted, non-rotating and full ceramic blades.

$$
\bar{\Omega}=\Omega \frac{b_{1} L^{4}}{a_{55}}, \quad \bar{\omega}_{j}^{2}=\omega_{j}^{2} \frac{b_{1} L^{4}}{a_{55}}
$$

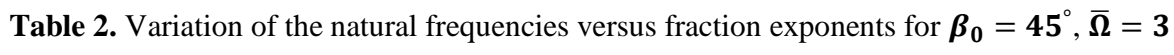

\begin{tabular}{ccccccc}
\hline \hline modes & & \multicolumn{5}{c}{ Volume fraction exponent } \\
\hline \hline \multirow{2}{*}{$\omega_{1}$} & Present & Ceramic $(k=0)$ & $k=1$ & $k=3$ & $k=5$ & Metal $(k=\infty)$ \\
& Ref [6] & 5.82 & 5.14 & 4.95 & 4.91 & 4.84 \\
$\omega_{2}$ & Present & 8.64 & 6.15 & 4.94 & 4.91 & 4.84 \\
& Ref [6] & 8.63 & 6.05 & 5.41 & 5.28 & 5.04 \\
$\omega_{3}$ & Present & 23.46 & 16.7 & 15.1 & 14.69 & 14.22 \\
& Ref [6] & 23.4 & 16.6 & 14.99 & 14.62 & 14.17 \\
\hline
\end{tabular}


Table 3. Material and geometrical properties of FG TWRB

\begin{tabular}{cc}
\hline \hline Properties & Value \\
\hline$E_{C}$ & $3.22 \times 10^{2} \mathrm{GPa}$ \\
$\rho_{C}$ & $2370 \mathrm{~kg} / \mathrm{m}^{3}$ \\
$v_{C}$ & 0.24 \\
$E_{m}$ & $2.08 \times 10^{2} \mathrm{GPa}$ \\
$\rho_{m}$ & $8166 \mathrm{~kg} / \mathrm{m}^{3}$ \\
$v_{m}$ & 0.32 \\
$R_{0}$ & $1.3 \mathrm{~m}$ \\
$L$ & $1.52 \mathrm{~m}$ \\
$l, d, h$ & $0.2570 .0827 \mathrm{~m}, 0.01654 \mathrm{~m}$ \\
\hline \hline
\end{tabular}

\subsection{Parametric Study}

This section presents free vibration, static, and buckling studies of functionally graded blade. The effects of volume fraction exponents $(k)$, dimensionless angular velocity $(\overline{\boldsymbol{\Omega}})$, pretwist angle $\left(\boldsymbol{\beta}_{\mathbf{0}}\right)$ on the dimensionless first two natural frequencies $\left(\bar{\omega}_{1}, \bar{\omega}_{2}\right)$, dimensionless chordwise and flapwise deformations $(\bar{u}, \bar{v})$ and dimensionless critical buckling load $(\bar{P})$ are compared for FG models presented in Table 4.

Some numerical results are presented to show the accuracy and reliability of the suggested technique and to perform a parametric study. The material characteristics used for FGM are listed in Table 3. In the result section, four models of FG blade are considered to study the static and dynamic performance of such structures.

Table 4. FG models of the rotary blade

\begin{tabular}{|c|c|}
\hline models & FG theory \\
\hline model_1 & Power-law \\
\hline model_2 & Trigonometric \\
\hline model_3 & Viola-Tornabene three-parameter \\
\hline model_4 & Viola-Tornabene three-parameter \\
\hline
\end{tabular}

Material profiles through the functionally graded thickness are illustrated in Figure 3. In Figure 3 the classical volume fraction profiles given in Equations (2)-(4) are presented as special cases of the general distribution laws (seen in Table 4) for volume fraction exponent $0.5,1$ and 3. Figure 3 shows the variations of the ceramic volume fraction $V_{f}$ through the thickness for different models.
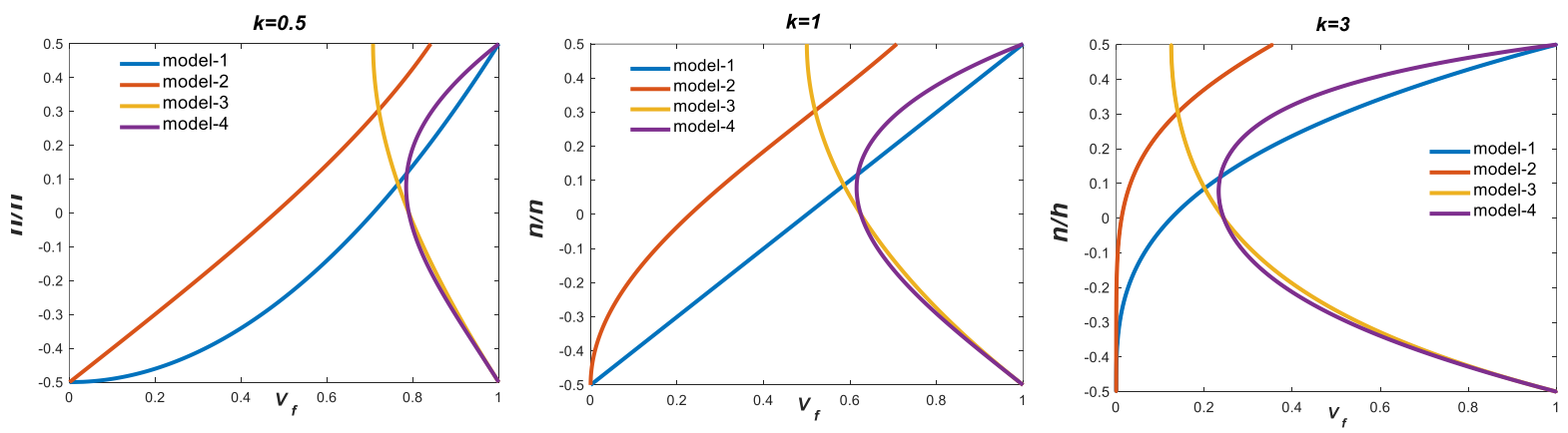

Figure 3. Variations of the ceramic volume fraction $V_{f}$ through the thickness for different models

To obtain a better perspective on the subject of the vibrational performance of FG blades, the variations of the first two dimensionless natural frequencies given in Equation (21) are examined for the various 
FG models. The first flapwise bending mode (1F) and the first chordwise bending mode (1C) as the 1st and 2nd natural modes are investigated, respectively.

A set of combined distributed loadings are considered in static analyses. A distributed load of $p_{y}$ in the negative $\mathrm{y}$-direction and $p_{x}$ in the positive $\mathrm{x}$-direction are applied to measure the resultant flapwise and chordwise deflections of the tip section of the TWRB. Non-dimensional chordwise and flapwise deflections are expressed as $\bar{u}=\frac{u_{0}}{h}$ and $\bar{v}=\frac{v_{0}}{h}$ in which $u_{0}$ and $v_{0}$ are tip chordwise and flapwise deflections of the TWRB, respectively, and $h$ is the wall thickness.

The dimensionless critical buckling loads $(\bar{P})$ (given in Equation (20)) of the FG TWRB subjected to unidirectional compressive loading in the $\mathrm{z}$-direction are predicted.

Considering Figure 4a-e one can compare diverse trends of the frequency variations $\left(\bar{\omega}_{1}, \bar{\omega}_{2}\right)$, chordwise and flapwise deflections $(\bar{u}, \bar{v})$ and critical buckling load $(\bar{P})$ versus variation of the angular velocity $\overline{\mathbf{\Omega}}$ for FG models defined in Table 4. In these figures, four FG distribution patterns and the constant values of $k=1, \boldsymbol{\beta}_{\mathbf{0}}=\mathbf{4 5}^{\circ}$ are considered in investigation. Figure $4 \mathrm{a}$ and $\mathrm{b}$ show a comparison among the first two natural frequencies of the TWRB with different FG models versus angular velocity. It is observed from Figure 4a and $\mathrm{b}$ that model_4 and model_3 (Viola-Tornabene three-parameter) provide higher natural frequencies compared to other profiles, respectively. It is due to the increasing the stiffness to mass ratio of the FG thin-walled beams is an effective way to improve the vibrational performance of the system. On the other hand, model_2 (Trigonometric) presents the lower natural frequencies. From Figure $4 \mathrm{a}$ and $\mathrm{b}$, it is found out that growing the angular velocity results in higher natural frequency in all FG profiles of the blade. This shows that the additional restoring force due to the centrifugal effect leads to an increase in the natural frequencies.

Among four FG profiles, the non-dimensional chordwise $(\bar{u})$ and flapwise $(\bar{v})$ deflections of the beam are examined and shown in Figure $4 \mathrm{c}$ and $\mathrm{d}$. The desirable criteria in the static analysis are to achieve the beam configuration with minimum deflection of the tip section of the beam subjected to the defined combined loads. With an increase in angular velocity the $\bar{u}$ and $\bar{v}$ deflections decrease significantly. At lower angular velocities $\overline{\mathbf{\Omega}}<2$ and $\overline{\mathbf{\Omega}}<1$, model_4 has minimum chordwise and flapwise deflections with the same amount of fraction exponent, respectively. But after mentioned specific velocities, the static characteristics of the blade significantly changes. At higher angular velocities $\overline{\mathbf{\Omega}}>2$ and $\overline{\mathbf{\Omega}}>1$, model_2 (Trigonometric) shows the best static performance for chordwise and flapwise deflections, respectively. It can be seen that the various FG patterns have an important role to improve the stiffness of the beam in various operation conditions. Angular velocity plays a promising role which reduces both $(\bar{u})$ and $(\bar{v})$ in all FG models. This reduction is more sensible in flapwise mode which is more affected by centrifugal force than chordwise mode.

Figure $4 \mathrm{e}$ shows the variation of the buckling load with respect to the variation of $\overline{\mathbf{\Omega}}$ and FG models. The maximum buckling load is identified at higher angular velocities due to the effect of centrifugal force. The centrifugal force effect increases the flapwise bending stiffness. The maximum and minimum buckling loads are seen in model_2 and model_4, respectively. 
Farsadi / Eskişehir Technical Univ. J. of Sci. and Tech. A-Appl. Sci. and Eng. 22 (3) - 2021

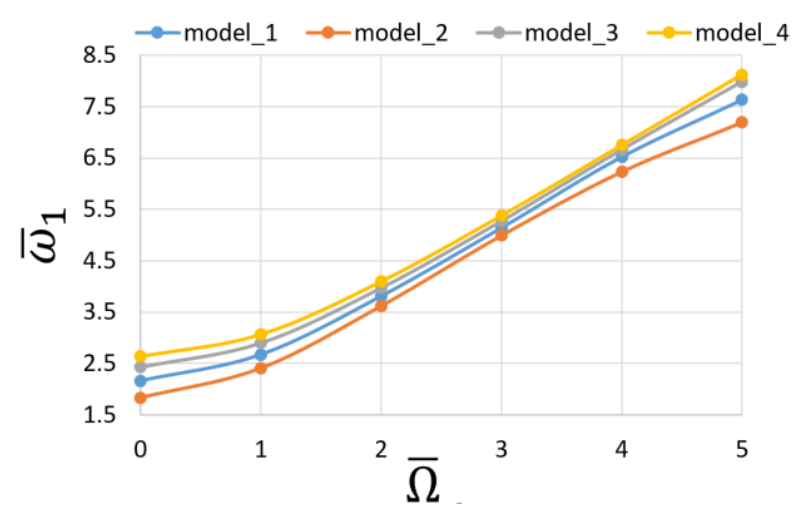

(a)

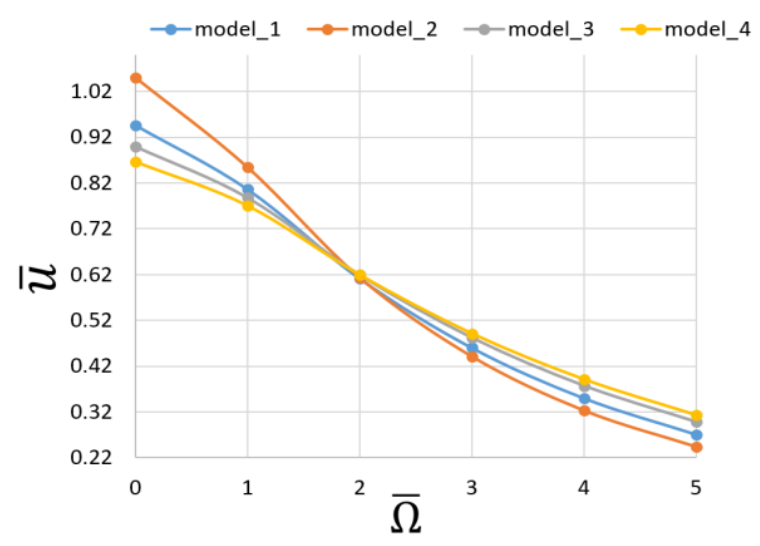

(c)

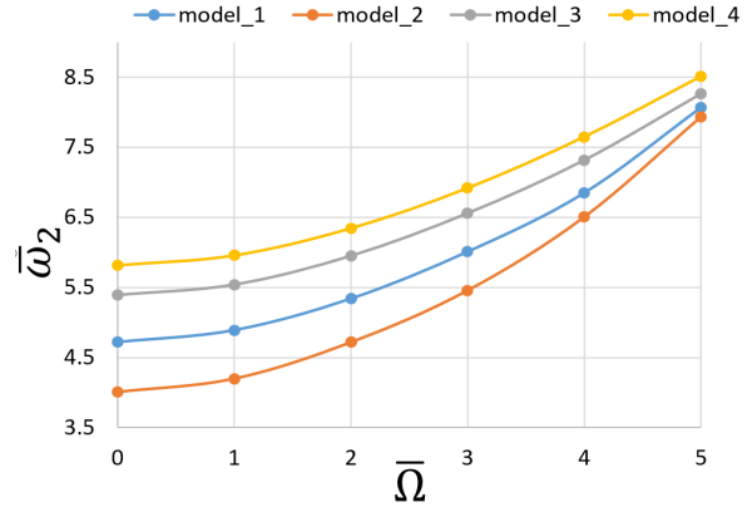

(b)

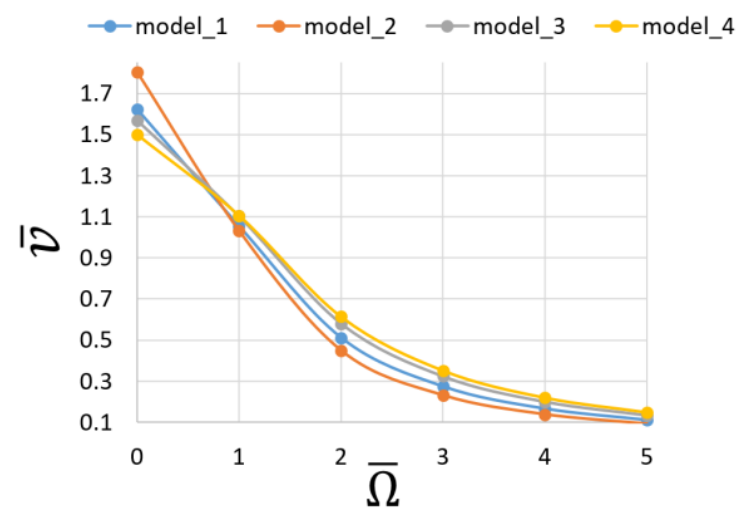

(d)

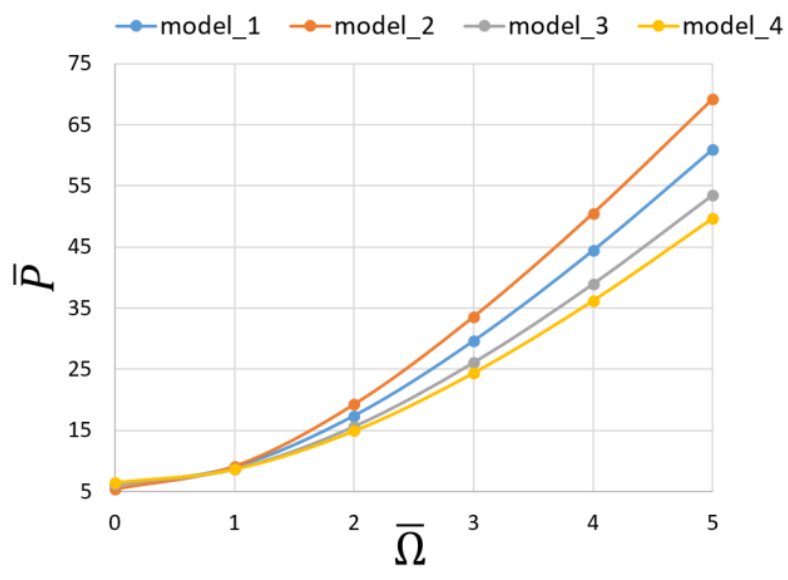

(e)

Figure 4. Variation of angular velocity $(\bar{\Omega})$ of the TWRB for various FG models versus a and b) $1^{\text {st }}$ and $2^{\text {nd }}$ natural frequency $\left(\bar{\omega}_{1}, \bar{\omega}_{2}\right)$, c and d) chordwise $(\bar{u})$ and flapwise $(\bar{v})$ deflections and e) critical buckling load

Figure 5a-e show the variation of the dynamic and static parameters of the blade against the variation of pretwist angle $\boldsymbol{\beta}_{\mathbf{0}}$ for various FG models. In these figures, four FG distribution patterns and the constant values of $k=1, \overline{\mathbf{\Omega}}=3$ are considered in investigation. The first and second natural frequencies have a little reduction as pretwist angle increases. 
The maximum $\bar{\omega}_{1}$ and $\bar{\omega}_{2}$ are identified in model_4 and minimum values are observed in model_2. The effect of $\boldsymbol{\beta}_{\mathbf{0}}$ variations on the non-dimensional chordwise $(\bar{u})$ and flapwise $(\bar{v})$ deflections of the TWRB are examined and shown in Figure $5 \mathrm{c}$ and d. The maximum $(\bar{u})$ and $(\bar{v})$ are identified in model_4 and minimum values are observed in model_2. It shows that model_2 presents the best static performance compared to the other FG functions.

With an increase in pretwist angle $\left(\boldsymbol{\beta}_{\mathbf{0}}\right)$ the $\bar{u}$ deflection continuously increases. It can be explained by the fact that increasing pretwist results in increasing the $a_{22}^{p}$ and $a_{44}^{p}$ chordwise shear and bending stiffness of the considered system. This trend is not exactly true for flapwise deflection. The $\bar{v}$ increases by increasing the $\boldsymbol{\beta}_{\mathbf{0}}$ until reaching the maximum values at $\boldsymbol{\beta}_{\mathbf{0}}=75^{\circ}$. After that, $\bar{v}$ smoothly decreases with an increase in $\boldsymbol{\beta}_{\mathbf{0}}$. The variation of the $a_{33}^{p}$ and $a_{55}^{p}$ with respect to $\boldsymbol{\beta}_{\mathbf{0}}$ have a significant impact on the flapwise deflections of the beam. Superscript $p$ denotes the stiffness coefficient in curvilinear coordinate. The maximum buckling load is identified at higher pretwist angles. In the case of the various FG models, the model_4 shows the worst buckling performance while the model_2 is spotted to has a strong appearance.

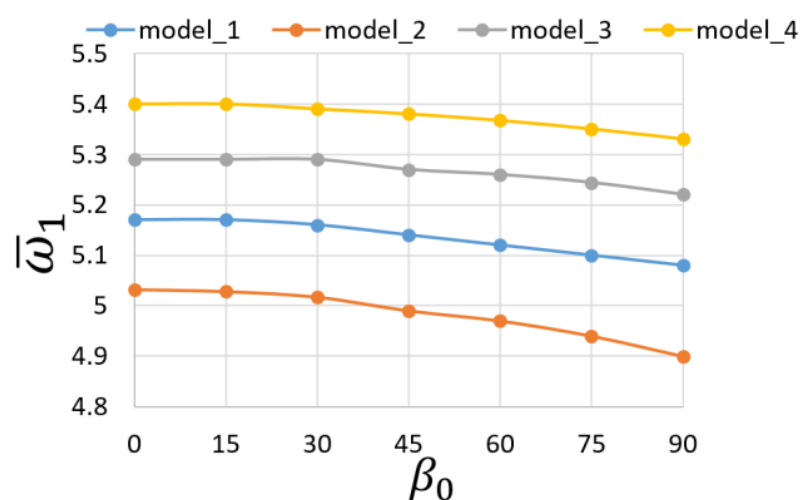

(a)

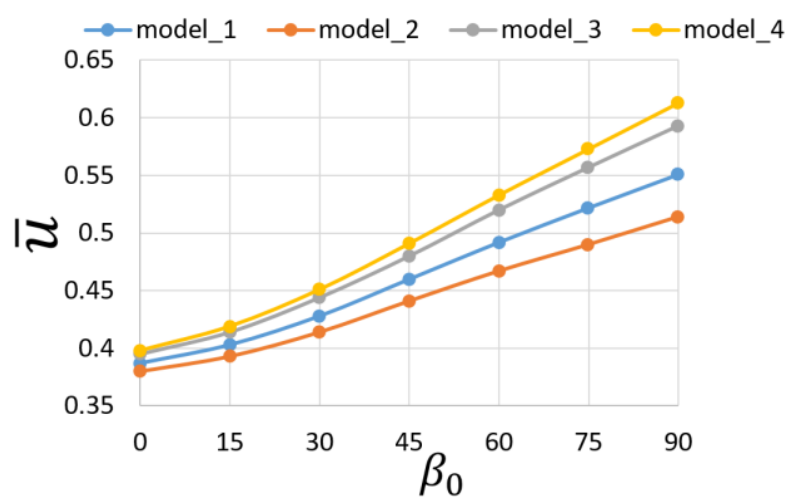

(c)

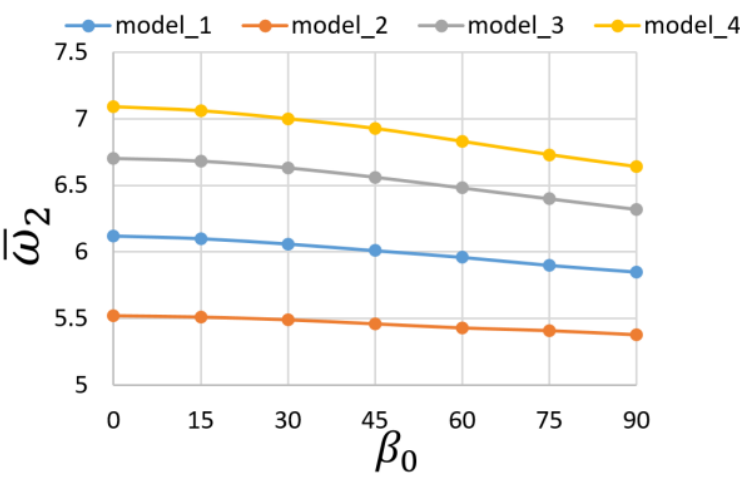

(b)

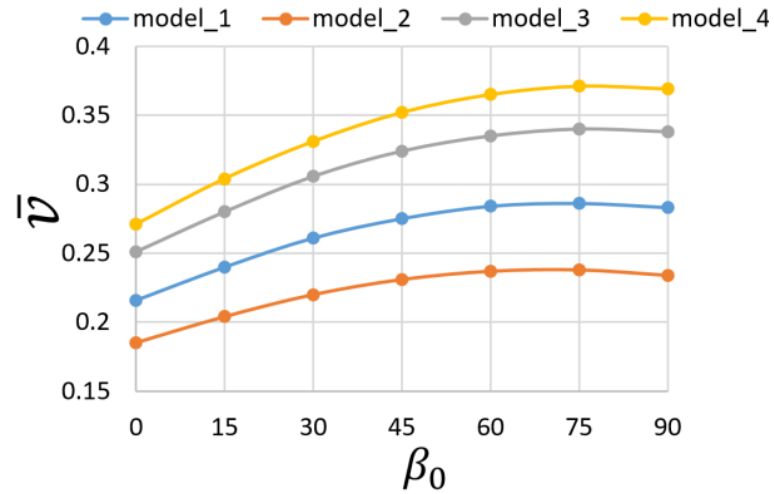

(d) 


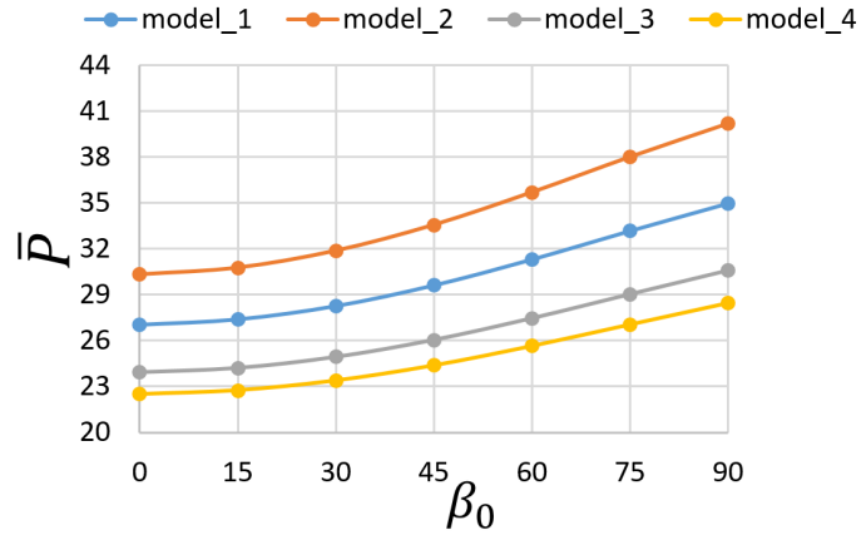

(e)

Figure 5. Variation of pretwist angle $\left(\beta_{0}\right)$ of the TWRB for various FG models versus a and $\left.b\right) 1^{\text {st }}$ and $2^{\text {nd }}$ natural frequency $\left(\bar{\omega}_{1}, \bar{\omega}_{2}\right)$, c and d) chordwise $(\bar{u})$ and flapwise $(\bar{v})$ deflections and e) critical buckling load

\section{CONCLUSION}

In the present work, the effect of various FG models on free vibration, static, and buckling performance of thin-walled rotating blades made of FGM are explored. Hamilton's principle is applied to extract the Euler-Lagrange governing equations of the dynamic system. The Extended Galerkin`s Method (EGM) is applied to solve the governing set of equations. The frequency, static chordwise and flapwise deflections, and buckling behavior of the FG TWRB are studied and compared for various FG models. According to the results, different FG models significantly affect the TWRB's natural frequencies, static deflections, and critical buckling load. The following conclusions are extracted from the study:

- The buckling and static analyses should be studied parallel to free vibration analysis to achieve the best structural performance.

- It can be found out that increasing the rotating speed predicts more natural frequency in all FG models of the blade. This means that the increase in the natural frequencies is due to the additional restoring force of the centrifugal effect.

- The first natural frequency varied smoothly by increasing pretwist angle except in model_2, while the second natural frequency has a reduction as pretwist angle increases, especially in model_3 and model_4.

- Angular velocity plays a promising role which reduces both $\overline{\boldsymbol{u}}$ and $\overline{\boldsymbol{v}}$ in all FG models. This reduction is more sensible in flapwise mode which is more affected by centrifugal force than chordwise mode. The minimum chordwise and flapwise deflections are seen in model_2 for $\overline{\mathbf{\Omega}} \geq 2$ and $\overline{\mathbf{\Omega}} \geq 1$, respectively.

- It is observed that at a given combined loading, the chordwise deflection of FG TWRB continuously increases as the pretwist angle increases in all FG models. The minimum chordwise deflection is seen in model_2. This trend is not true for flapwise deflection. The $\bar{v}$ increases by increasing the $\boldsymbol{\beta}_{\mathbf{0}}$ until reaching the maximum value. After that, $\bar{v}$ smoothly decreases with an increase in $\boldsymbol{\beta}_{\mathbf{0}}$. The minimum flapwise deflection is seen in model_2.

- The trend of the continuous increase in the buckling load is seen for angular velocity and pretwist growth.

\section{CONFLICT OF INTEREST}

The author stated that there are no conflicts of interest regarding the publication of this article. 


\section{REFERENCES}

[1] Thai C H, Kulasegaram, S, Tran LV, Nguyen-Xuan H. Generalized shear deformation theory for functionally graded isotropic and sandwich plates based on isogeometric approach. Computers \& Structures, 2014; 141: 94-112.

[2] Wang YQ, Zu JW. Vibration behaviors of functionally graded rectangular plates with porosities and moving in thermal environment. Aerospace Science and Technology, 2017; 69: 550-562.

[3] Yu TT, Yin S, Bui TQ, Hirose S. A simple FSDT-based isogeometric analysis for geometrically nonlinear analysis of functionally graded plates. Finite Elements in Analysis and Design, 2015; 96: 1-10.

[4] Farsadi T, Rahmanian M, Kurtaran H. Nonlinear analysis of functionally graded skewed and tapered wing-like plates including porosities: A bifurcation study. Thin-Walled Structures, 2021; 160: 107341.

[5] Mahamood RM, Akinlabi ET. Types of functionally graded materials and their areas of application. In Functionally Graded Materials, 2017: 9-21, Springer, Cham.

[6] Librescu L, Oh SY, Song O. Thin-walled beams made of functionally graded materials and operating in a high temperature environment: vibration and stability. Journal of Thermal Stresses 2005; 28 (6-7): 649-712.

[7] Oh SY, Librescu L, Song O. Thermoelastic modeling and vibration of functionally graded thinwalled rotating blades. AIAA journal, 2003; 41(10): 2051-2061.

[8] Bahaadini R, Saidi AR. Aeroelastic analysis of functionally graded rotating blades reinforced with graphene nanoplatelets in supersonic flow. Aerospace Science and Technology, 2018; 80: 381-391.

[9] Piovan MT, Machado SP. Thermoelastic dynamic stability of thin-walled beams with graded material properties. Thin-walled structures. 2011; 49(3): 437-447.

[10] Latalski J, Warminski J. Dynamics of rotating thin-walled cantilever composite beam excited by translational motion. Procedia Engineering. 2016; 144: 1039-1046.

[11] Sina SA, Ashrafi MJ, Haddadpour H, Shadmehri F. Flexural-torsional vibrations of rotating tapered thin-walled composite beams. Proceedings of the Institution of Mechanical Engineers, Part G: Journal of Aerospace Engineering, 2011; 225(4): 387-402.

[12] Oh Y, Yoo HH. Vibration analysis of rotating pretwisted tapered blades made of functionally graded materials. International Journal of Mechanical Sciences, 2016; 119: 68-79.

[13] Fazelzadeh SA, Hosseini M. Aerothermoelastic behavior of supersonic rotating thin-walled beams made of functionally graded materials. Journal of Fluids and Structures, 2007; 23(8): 12511264.

[14] Fazelzadeh SA, Malekzadeh P, Zahedinejad P, Hosseini M. Vibration analysis of functionally graded thin-walled rotating blades under high temperature supersonic flow using the differential quadrature method. Journal of Sound and Vibration, 2007; 306(1-2): 333-348. 
[15] Oh SY, Song O, Librescu L. Effects of pretwist and presetting on coupled bending vibrations of rotating thin-walled composite beams. International Journal of Solids and Structures, 2003; 40(5): $1203-1224$.

[16] Oh SY, Librescu L, Song O. Vibration of turbomachinery rotating blades made-up of functionally graded materials and operating in a high temperature field. Acta Mechanica, 2003; 166(1-4): 6987.

[17] Maalawi K. Functionally graded bars with enhanced dynamic performance. Journal of Mechanics of Materials and Structures, 2011; 6(1): 377-393.

[18] Farsadi T. Enhancement of static and dynamic performance of composite tapered pretwisted rotating blade with variable stiffness. Journal of Vibration and Acoustics, 2021; 143(2).

[19] Farsadi T, Şener Ö, Kayran A. Free vibration analysis of uniform and asymmetric composite pretwisted rotating thin walled beam. In ASME International Mechanical Engineering Congress and Exposition, American Society of Mechanical Engineers 2017 November; 58349: V001T03A016).

[20] Librescu L, Song O. Thin-walled composite beams: theory and application. Springer Science \& Business Media, 2006; 131.

[21] Asadi D, Farsadi T, Kayran A. Flutter Optimization of a Wing-Engine System with Passive and Active Control Approaches. AIAA Journal, 2021; 1-19. 